\title{
Laser-ultrasonic Monitoring of Austenite Recrystallization in C-Mn Steel
}

\author{
Ali SMITH, ${ }^{1,2)}$ Silvio E. KRUGER, ${ }^{3)}$ Jilt SIETSMA ${ }^{2)}$ and Sybrand van der ZWAAG ${ }^{4)}$ \\ 1) Netherlands Institute for Metals Research, 2628 CD Delft, the Netherlands. \\ 2) Department of Materials Science \& Engineering, Delft University of Technology, 2628 CD Delft, the Netherlands. \\ 3) IMI-National Research Council of Canada 75 Boul. de Mortagne, Boucherville, Quebec, Canada, J4B 6Y4. \\ 4) Faculty of Aerospace Engineering, Delft University of Technology, $2629 \mathrm{HS}$ Delft, the Netherlands.
}

(Received on November 14, 2005; accepted on May 24, 2006)

\begin{abstract}
The static recrystallization kinetics of hot deformed austenite were characterised by a combination of insitu laser-ultrasonics and stress relaxation measurements. Samples were deformed in compression at temperatures of $850^{\circ} \mathrm{C}$ and $900^{\circ} \mathrm{C}$. The applied strain was varied between 0.2 and 0.5 , whilst the strain rates used were $0.1 \mathrm{~s}^{-1}$ and $0.5 \mathrm{~s}^{-1}$. At various points during annealing samples were quenched and analysed by optical microscopy.

During recovery prior to recrystallization the ultrasonic velocity change and attenuation were almost constant. During recrystallization, the velocity change and attenuation decreased for the conditions imposed. The decrease in velocity change was mostly due to a change in the overall texture. The decrease in attenuation could be explained by a change in grain size. Unfortunately, the additional effect of dislocation damping on attenuation could not be quantified.

Comparison of the stress relaxation kinetics with the observed decrease in ultrasonic velocity change and attenuation generally showed good agreement.

From the attenuation measurements the grain sizes in the recrystallised structure for various applied strains were calculated. Comparison with grain sizes determined from optical microscopy showed reasonable agreement considering the accuracy of the attenuation measurements and the assumptions made.
\end{abstract}

KEY WORDS: laser ultrasonics; stress relaxation; recrystallization; grain size.

\section{Introduction}

Two of the most important phenomena that occur during hot rolling of steels in the austenitic state are static recrystallization and subsequent grain growth. These processes determine the austenite grain size development in between mill stands (and hence the rolling forces required to induce the prescribed deformation), as well as the starting state for the final austenite to ferrite phase transformation. Thus an accurate description of static recrystallization and grain growth in the austenitic state is highly relevant for process control.

Up to now the recrystallization kinetics in austenite are best studied by using the established in-situ stress relaxation technique. ${ }^{1-4)}$ In this technique samples can also be quenched, etched and analysed by optical microscopy to reveal the austenite grain size just before quenching. This allows assessment of the evolution in grain size during recrystallization and grain growth. Whilst being a valuable technique, stress relaxation combined with quenching is destructive and thus cannot be employed directly to monitor recrystallization and grain growth during industrial processing. In recent years the use of non-contact laser-ultrasonics has been demonstrated as a promising technique to overcome this barrier. Ultrasonic studies have been per- formed to assess recrystallization kinetics ${ }^{5-8)}$ and grain size. $^{9-12)}$ In addition, online mill trials have already been performed during the processing of steel strip, ${ }^{13}$ sheet, ${ }^{14)}$ and an ultrasonic system has been reported to be continuously operating for a few years to monitor tube thickness and austenite grain size. ${ }^{15)}$

Whilst much progress has been made in the field of ultrasonics in metallurgy, there is still a lack of ultrasonic studies of annealing phenomena following hot deformation of steels. The studies mentioned earlier were performed on samples annealed after cold deformation only. In addition, those studies in which the grain size was evaluated did not concern the grain size evolution following recrystallization. Thus, if this technique is to be used for online monitoring of recrystallization and grain growth in steels, then further study is required.

The present investigation focuses on recrystallization of austenite following hot deformation. Measurements of ultrasonic velocity and attenuation have been made during annealing for a variety of deformation conditions and temperatures. Stress relaxation measurements were made simultaneously, since this would provide a useful comparison and greatly aid the interpretation of the results from the laser-ultrasonics technique. The steel chosen for the study was a plain carbon manganese steel, its recrystallization be- 
haviour has been well characterized, ${ }^{2,3)}$ allowing easier interpretation of the ultrasonics data. Secondly, for this steel composition samples could be water quenched to form a fully martensitic structure. Thus prior austenite grain boundaries can be shown following etching of quenched samples. This allowed the progress of recrystallization to be monitored optically as well.

Finally, the results obtained are linked to the recrystallization process and the final austenite grain size.

\section{Effect of Microstructure on Ultrasonic Velocity and Attenuation}

Microstructural features cause changes in velocity and attenuation of ultrasonic waves. In a paramagnetic phase such as austenite, the ultrasonic velocity mainly depends on texture, ${ }^{5,6)}$ dislocation structure ${ }^{16-18)}$ and on scattering effects in general. Scattering is caused by acoustic inhomogeneities, like grain boundaries, porosity, cracks, etc. In this study only grain scattering needs to be considered.

The attenuation of ultrasonic waves is due to grain scattering and absorption effects, ${ }^{16-19)}$ the latter (in a paramagnetic phase) being mainly due to the dislocation structure.

Thus the ultrasonic velocity and attenuation during annealing should be due to a combination of the above effects, depending on the deformation and temperature conditions. In the following sections the theories for each of these effects are discussed.

\subsection{Texture Effects}

In the presence of texture, the longitudinal ultrasonic velocity $v_{\mathrm{Lx}}$ is given by ${ }^{5}$ :

$$
v_{\mathrm{Lx}}=\sqrt{\frac{1}{\Lambda}\left(\lambda+2 \mu+\frac{32 \sqrt{2}}{35} \pi^{2} c W_{400}\right)}
$$

where $\Lambda$ is the density of the material, $\lambda, \mu$ and $c$ are single crystal elastic constants and $W_{400}$ is a texture coefficient. This coefficient comes from a series expansion of the crystallographic orientation distribution function. For an orthorhombic aggregate of cubic crystallites $W_{400}$ is one of the lowest order non-zero texture coefficients in the Roe notation. ${ }^{5,14)}$

During isothermal annealing the elastic constants and density can be assumed constant. In most metals the texture term $(32 \sqrt{2} / 35) \pi^{2} c W_{400}$ is much smaller than the constant term $(\lambda+2 \mu)$ and Eq. (1) can be simplified to

$$
v_{\mathrm{Lx}}=d+e W_{400}
$$

where $d$ and $e$ are constants. Thus, according to Eq. (2) the velocity is linearly related to the texture coefficient. Since the texture changes during recrystallization, time dependent changes in the ultrasonic velocity during annealing provide information about the kinetics of the recrystallization process.

\subsection{Grain Scattering Effects}

Grain scattering affects both the velocity and attenuation of ultrasonic waves. The relationships between velocity and grain size or attenuation and grain size depend on the ratio of the ultrasonic wavelength in the material $\lambda_{u}$ to the grain size $D_{\mathrm{g}}$. For the experimental results presented here the ultrasonic wavelength in the material is around an order of magnitude larger than the average deformed grain size. Thus, assuming the Rayleigh regime $\left(\lambda_{\mathrm{u}} \gg D_{\mathrm{g}}\right)$, the group velocity $v_{\mathrm{LGg}}$ and attenuation $\alpha_{\mathrm{Lg}}$ for longitudinal waves are given by ${ }^{20,10)}$.

$$
\begin{aligned}
& v_{\mathrm{LGg}} \\
& =\frac{v_{\mathrm{L}}}{\left(1+0.00666\left(\frac{A}{\Lambda v_{\mathrm{L}}^{2}}\right)^{2}\left(14+\frac{21 \kappa^{2}}{k^{2}}+\frac{6}{5}\left(\frac{106}{7}+\frac{6 \kappa^{2}}{7 k^{2}}+\frac{21 \kappa^{4}}{k^{4}}\right)\left(k D_{\mathrm{g}}\right)^{2}\right)\right)} \\
& \alpha_{\mathrm{Lg}}=S_{\mathrm{L}} D_{\mathrm{g}}^{3} f^{4}
\end{aligned}
$$

where $A$ is a single-crystal anisotropy factor, $f$ is the ultrasonic frequency and $S_{\mathrm{L}}$ is the scattering factor for longitudinal waves.

The wave numbers $\kappa$ and $k$ in Eq. (3) are given by ${ }^{20)}$ :

$$
\begin{gathered}
\kappa=\frac{2 \pi f}{v_{\mathrm{s}}} \\
k=\frac{2 \pi f}{v_{\mathrm{L}}}
\end{gathered}
$$

where $v_{\mathrm{s}}$ is the shear wave velocity.

For Eq. (4), $S_{\mathrm{L}}$ is given by ${ }^{21)}$ :

$$
S_{\mathrm{L}}=\frac{0.0308 \pi^{4} \mu_{\mathrm{a}}^{2}}{\Lambda^{2} v_{\mathrm{L}}^{2}}\left(\frac{2}{v_{\mathrm{L}}^{5}}+\frac{3}{v_{\mathrm{s}}^{5}}\right)
$$

where $\mu_{\mathrm{a}}$ is related to the anisotropy of the grain and is defined as:

$$
\mu_{\mathrm{a}}=c_{11}-c_{12}-2 c_{44}
$$

where $c_{11}, c_{12}$ and $c_{44}$ are the elastic constants of the cubic crystal.

It should be noted that in the above equations the grains are assumed to be spherical. Thus Eqs. (3) and (4) should be particularly applicable for an undeformed or fully recrystallized microstructure, where the grain shape can be assumed roughly spherical.

\subsection{Dislocation Damping}

The effect of dislocations on velocity and attenuation has been treated by Granato and Lucke. ${ }^{22)}$ In their model, a dislocation is modelled as a string, which vibrates between pinning points. Forced vibration of the dislocation occurs by the interaction of the dislocation with the ultrasonic waves. In addition, the motion of the dislocation is inhibited by a drag force. The magnitude of this depends on a combination of thermal vibrations (phonons), electrons, and impurity atoms. 
Assuming strain-amplitude independent damping, in the lower frequency range, the relative velocity change $\left(\left(v_{\mathrm{d}}(\rho)-v_{\mathrm{d}}(0)\right) / v_{\mathrm{d}}(0)\right)$ and attenuation $\alpha_{\mathrm{d}}($ in $\mathrm{dB} / \mathrm{s})$ due to the presence of dislocations with density $\rho$ and average pinning point separation $L$, are given by the equations ${ }^{22)}$.

$$
\begin{gathered}
\left(\frac{v_{\mathrm{d}}(\rho)-v_{\mathrm{d}}(0)}{v_{\mathrm{d}}(0)}\right)=\frac{4 G b^{2} \rho L^{2}}{\pi^{4} C} \\
\alpha_{\mathrm{d}}=\frac{16 G B b^{2} f^{2} \rho L^{4}}{0.115 \pi^{4} C^{2}} \ldots \ldots .
\end{gathered}
$$

where $v_{\mathrm{d}}(\rho)$ is the velocity due to dislocations, $v_{\mathrm{d}}(0)$ is the velocity when there are no dislocations, $G$ is the shear modulus, $b$ is the Burgers vector, $C$ is the dislocation line tension and $B$ is the damping constant.

As can be seen from Eqs. (9), (10), the velocity and attenuation are dependent on both the dislocation density and the pinning point separation.

\subsection{Total Effect of Austenitic Microstructure}

To summarise, the overall attenuation $\alpha_{\mathrm{T}}$ and velocity change $\left(\Delta v / v_{0}\right)_{\mathrm{T}}$ expected from a particular experimental measurement can be given by:

$$
\alpha_{\mathrm{T}}=\alpha_{\mathrm{d}}+\alpha_{\mathrm{Lg}}
$$

and

$$
\begin{aligned}
\left(\frac{\Delta v}{v_{0}}\right)_{\mathrm{T}}= & \left(\frac{v_{\mathrm{Lx}}\left(W_{400}\right)-v_{\mathrm{Lx}}(0)}{v_{\mathrm{Lx}}(0)}\right)+\left(\frac{v_{\mathrm{LGg}}\left(D_{\mathrm{g}}\right)-v_{\mathrm{LGg}}(\infty)}{v_{\mathrm{LGg}}(\infty)}\right) \\
& +\left(\frac{v_{\mathrm{d}}(\rho)-v_{\mathrm{d}}(0)}{v_{\mathrm{d}}(0)}\right)
\end{aligned}
$$

where $v_{\mathrm{Lx}}(0)$ and $v_{\mathrm{LGg}}(\infty)$ are the velocities in the absence of texture $\left(W_{400}=0\right.$, Eq. (1)) and grain scattering $\left(D_{\mathrm{g}}=\infty\right.$, Eq. (3)) respectively. In obtaining $v_{0}$ the minimum values observed in the time scale of each experiment were used.

\section{Experimental}

The composition of the $\mathrm{C}-\mathrm{Mn}$ steel used in the experiments is given in Table 1. Cylindrical samples were machined from rolled plate, with diameter $10 \mathrm{~mm}$ and length $12 \mathrm{~mm}$. The samples were machined with the axis parallel to the rolling direction.

Figure 1(A) shows the experimental set-up used for the ultrasonic measurements. A Nd: YAG laser (pulse energy of $200 \mathrm{~mJ}$, pulse duration of $5 \mathrm{~ns}$, wavelength of $532 \mathrm{~nm}$ ) is used to generate ultrasonic waves in the deformed sample. The ultrasonic waves propagate through the sample and are reflected off the back surface. Each time the waves reach the original sample surface a small displacement of the surface occurs. These "echoes" are detected by a second laser (pulse duration of $50 \mu \mathrm{s}$, peak power of $1 \mathrm{~kW}$, wavelength of $1064 \mathrm{~nm}$ ) coupled to a confocal Fabry-Perot interferometer. The signals are digitised and recorded for further processing. Figure 1(B) shows an example of a signal obtained
Table 1. Steel composition shown in [wt $\%$ ].

\begin{tabular}{|c|c|c|c|c|}
\hline $\mathbf{C}$ & $\mathbf{M n}$ & $\mathbf{S i}$ & $\mathbf{A l}$ & $\mathbf{F e}$ \\
\hline 0.19 & 1.46 & 0.44 & 0.03 & Bal. \\
\hline
\end{tabular}

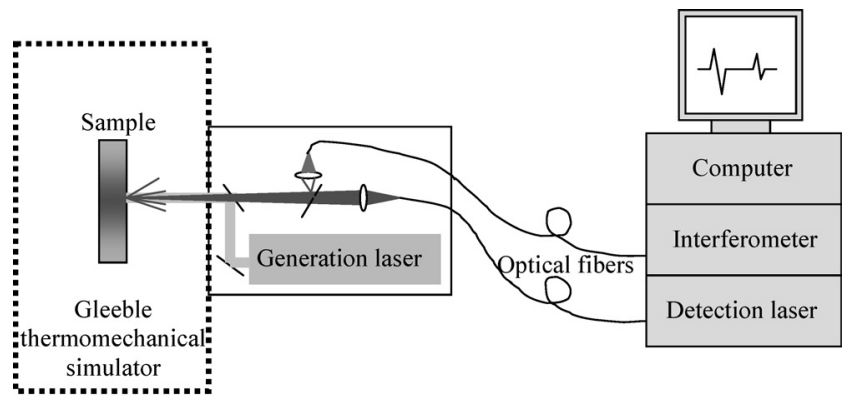

A)

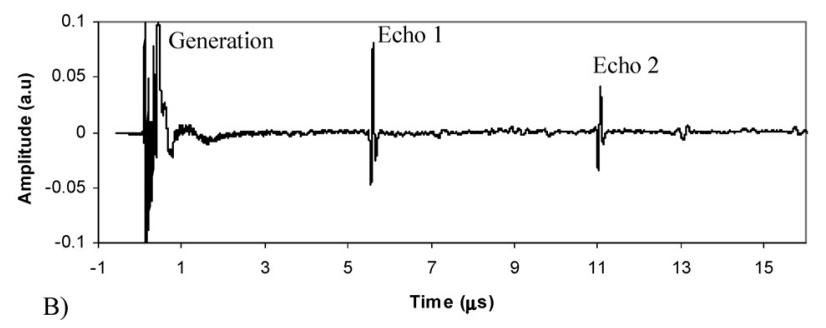

Fig. 1. (A) Laser ultrasonic monitoring apparatus. (B) Example of signal obtained.

during annealing. The signals are used to calculate the ultrasonic velocity and attenuation. The velocity was determined by the ratio of the distance travelled through the material (twice the sample diameter) to the time delay between generation and the first echo. The attenuation was determined by the ratio of the amplitude spectra of the first echo to the second echo, resulting in an attenuation versus frequency curve. Finally, this curve should be corrected for diffraction effects to provide the true attenuation of the material. However due to the sample geometry present after deformation (barreled cylinder), a diffraction correction could not be made.

To investigate the annealing kinetics, a thermo-mechanical simulator coupled with a laser-ultrasonic monitoring device was used. Each test comprised of three stages. Firstly, the desired starting microstructure was made via austenitisation at $1100^{\circ} \mathrm{C}$ for $3 \mathrm{~min}$, using electrical resistance heating under vacuum. The samples were cooled to $679^{\circ} \mathrm{C}$ (in the two-phase region) and held for $10 \mathrm{~min}$. (This was done for future comparison with intercritical deformation tests.) The samples were reheated to the desired test temperature in the austenite phase and held for $5 \mathrm{~min}$.

In the second stage, the samples were deformed in uniaxial compression mode. Lubrication was provided via graphite paste. To protect against carbon pick-up during the test, tantalum sheets were used as a protective layer between the sample and the lubricant. In addition, just before deformation the chamber was filled with argon, since this provided better conditions for the generation of ultrasonic waves.

Finally, in the third stage the annealing kinetics after deformation was monitored via the stress relaxation and laserultrasonics techniques. In the stress relaxation technique the stress required to maintain a constant strain in the sample 
was recorded. In the laser-ultrasonics technique, the ultrasonic signals were recorded for later calculation of velocity and attenuation.

To investigate the microstructural development during annealing, selected samples were water-quenched after different annealing times. To provide less interference with the ultrasonic measurements, the quenching was carried out in a separate (identical) thermo-mechanical simulator for the same experimental conditions. Samples were quenched at a rate of approximately $200^{\circ} \mathrm{C} / \mathrm{s}$. Quenched samples were analysed by optical microscopy, after suitable metallographic preparation.

\section{Results}

\subsection{Microstructural Evolution}

Figures 2-4 show the microstructures obtained from quenched samples for various applied strains.

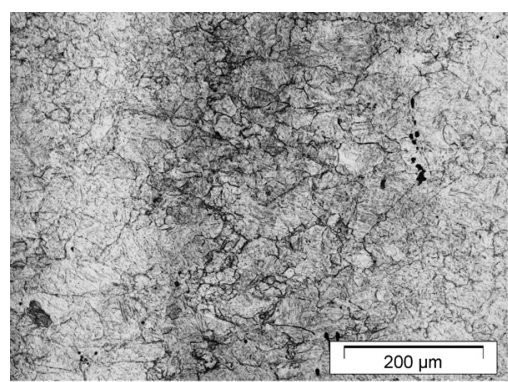

A)

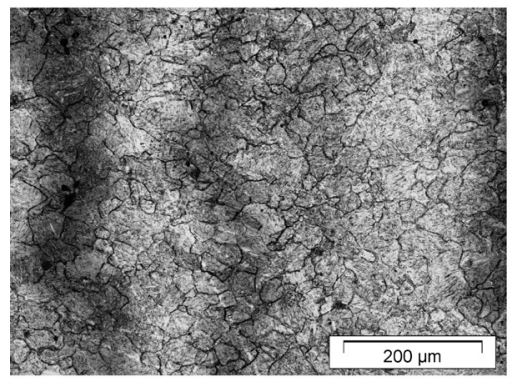

C)

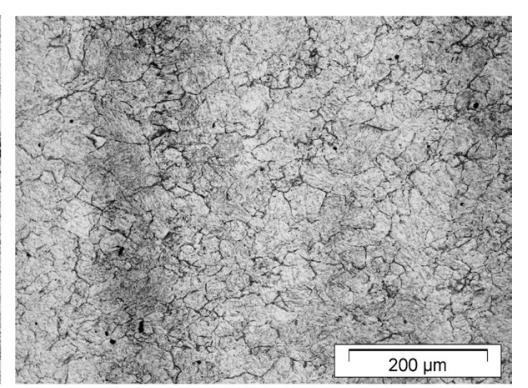

B)

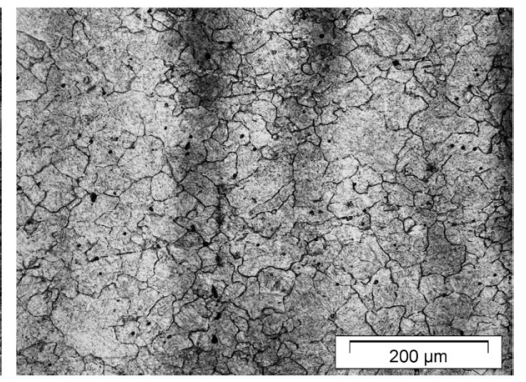

D)

Fig. 2. Optical Micrographs showing austenite microstructures during annealing at $850^{\circ} \mathrm{C}$ with strain $=0.2$ and strain rate $=0.5 \mathrm{~s}^{-1}$. (A) $3 \mathrm{~s}$, (B) $10 \mathrm{~s}$, (C) $100 \mathrm{~s}$, (D) $250 \mathrm{~s}$.

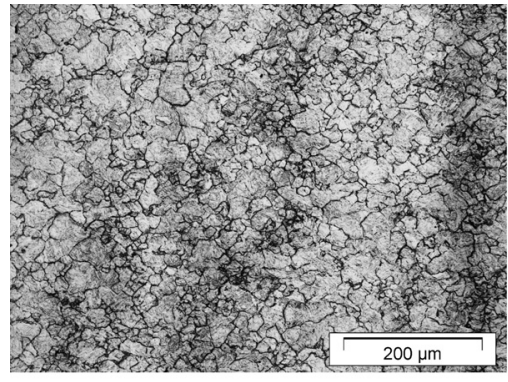

A)

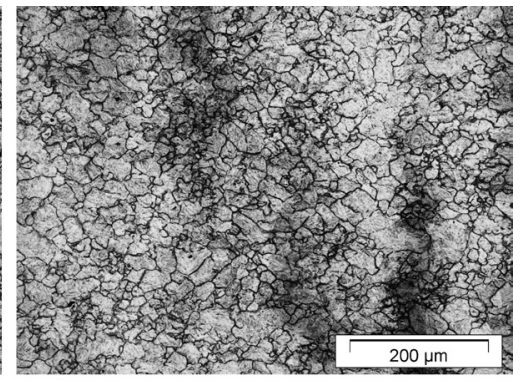

B)

Fig. 3. Optical micrographs after annealing at $850^{\circ} \mathrm{C}$. Strain rate $=0.5 \mathrm{~s}^{-1}$ and strain $=0.35$. (A) $50 \mathrm{~s}$, (B) $100 \mathrm{~s}$.

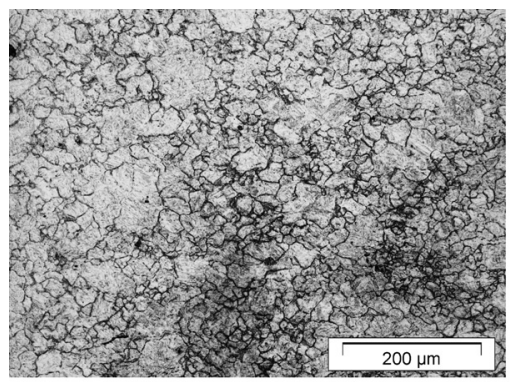

A)

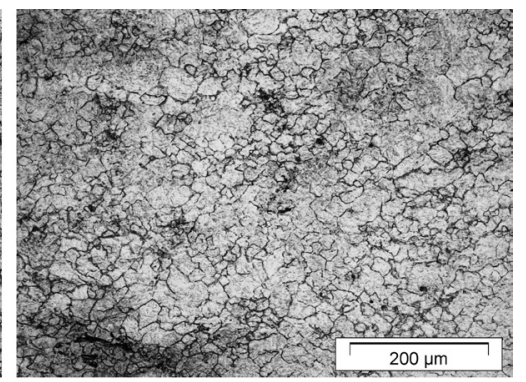

B)

Fig. 4. Optical micrographs after annealing at $850^{\circ} \mathrm{C}$. Strain rate $=0.5 \mathrm{~s}^{-1}$ and strain $=0.5$. (A) $50 \mathrm{~s}$, (B) $100 \mathrm{~s}$. 
Table 2. Grain sizes as determined by optical microscopy.

\begin{tabular}{|c|c|c|}
\hline Strain & Annealing time (s) & Grain size $(\boldsymbol{\mu m})$ \\
\hline 0.2 & 100 & 40 \\
\hline \multirow{2}{*}{0.35} & 50 & 25 \\
\cline { 2 - 3 } & 100 & 24 \\
\hline \multirow{2}{*}{0.5} & 50 & 21 \\
\cline { 2 - 3 } & 100 & 20 \\
\hline
\end{tabular}

For an applied strain of 0.2 it can be seen in Fig. 2 that after 3 and $10 \mathrm{~s}$ the structures appear to be partially recrystallised, with the degree of recrystallization after $10 \mathrm{~s}$ annealing appearing stronger. After $100 \mathrm{~s}$ the structure appears to be fully recrystallized, with some large grains visible, indicating grain growth. The structure after $250 \mathrm{~s}$ appears to be very similar to that after $100 \mathrm{~s}$, again with a few large grains.

For higher strains, i.e. 0.35 in figure 3 and 0.5 in Fig. 4, fully recrystallized structures can be seen with little difference between $50 \mathrm{~s}$ and $100 \mathrm{~s}$ anneals. There is again some evidence for grain growth. This is most obvious for Fig. 4, where a few large grains are visible.

A quantitative analysis of the grain size evolution is presented in Table 2. The grain size was measured in the centre of each sample, with a minimum of 300 grains being assessed for each sample. The average grain size presented in Table 2 is the average equivalent circle diameter.

As can be seen, the average grain size decreases with applied strain increasing. For strains of 0.35 and 0.5 , the average grain size remains almost constant between 50 and $100 \mathrm{~s}$ annealing.

\subsection{Ultrasonics and Stress Relaxation: Effect of Strain}

Figure 5 shows the effect of applied strain on the ultrasonic velocity change and stress relaxation. The stress relaxation kinetics exhibits three distinct stages. ${ }^{3)}$ Firstly there is an almost linear decay in stress as a function of $\log (t)$, which is generally attributed to recovery of austenite. ${ }^{3)}$ Secondly there is a sharper change in stress due to recovery in combination with recrystallization, in line with changes shown in Fig. 2. If the rate of reduction in stress is defined as the reciprocal of the time for $50 \%$ reduction, then the stress relaxation rate observed during recrystallization appears to increase with imposed strain. Finally, when recrystallization is complete, a smaller decrease in stress is observed. This has previously been attributed to the stress relaxation of the recrystallized austenite by recovery. ${ }^{3)}$ Another factor that could influence this stress decrease is grain growth. Although some grain growth during relaxation was suggested by the micrographs, Table 2 indicated that the average grain size remained constant. Thus, in this study the stress decrease following recrystallization is not expected to be influenced by grain growth.

The ultrasonic velocity changes shown in Fig. 5 also reveal three stages: firstly, for short times, where only recovery occurs, the velocity change remains constant or decreases only slightly. Secondly, when recrystallization occurs as well, the velocity change drops sharply, with a rate being larger for higher strains. In the third stage, the velocity change appears to tend to a minimum value, i.e. $v_{0}$ in Eq. (12).

Figure 6 shows the attenuation during recovery and recrystallization for different applied strains. The data shown

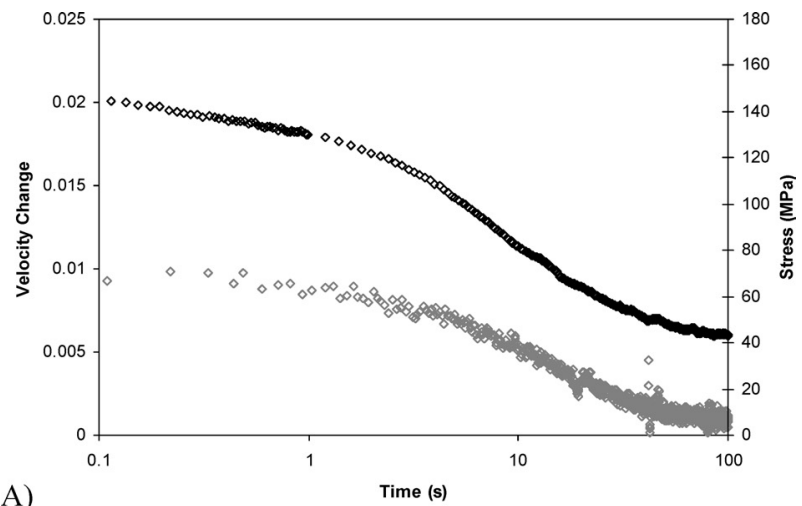

A)

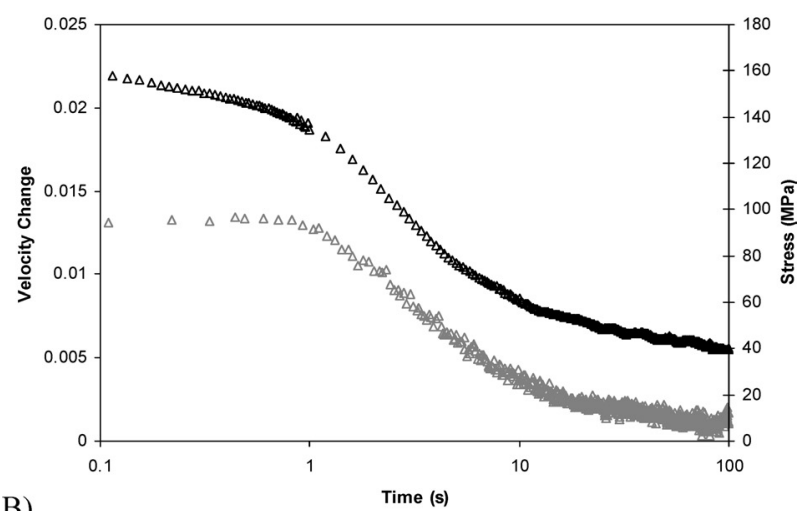

B)

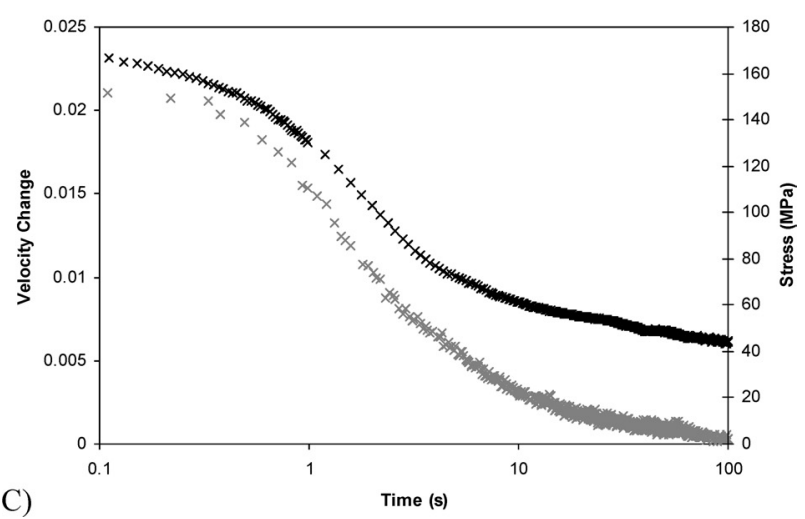

Fig. 5. Effect of strain on ultrasonic velocity (in grey) and stress relaxation (in black). $T=850^{\circ} \mathrm{C}$, strain rate $=0.5 \mathrm{~s}^{-1}$. (A) Strain $=0.2,(B)$ strain $=0.35,(C)$ strain $=0.5$.

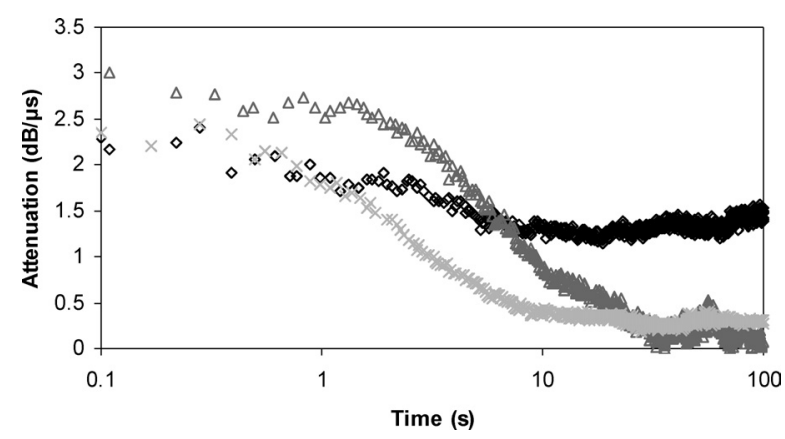

Fig. 6. Effect of strain on ultrasonic attenuation at $8 \mathrm{MHz}$. Strain rate $=0.5 \mathrm{~s}^{-1}, T=850^{\circ} \mathrm{C}$. Black diamonds indicate strain of 0.2 , dark grey triangles indicate strain of 0.35 and light grey crosses indicate strain of 0.5 .

is for a frequency of $8 \mathrm{MHz}$. For other frequencies the results showed the same trends. For lower frequencies changes in attenuation are less clearly observed, whilst for 


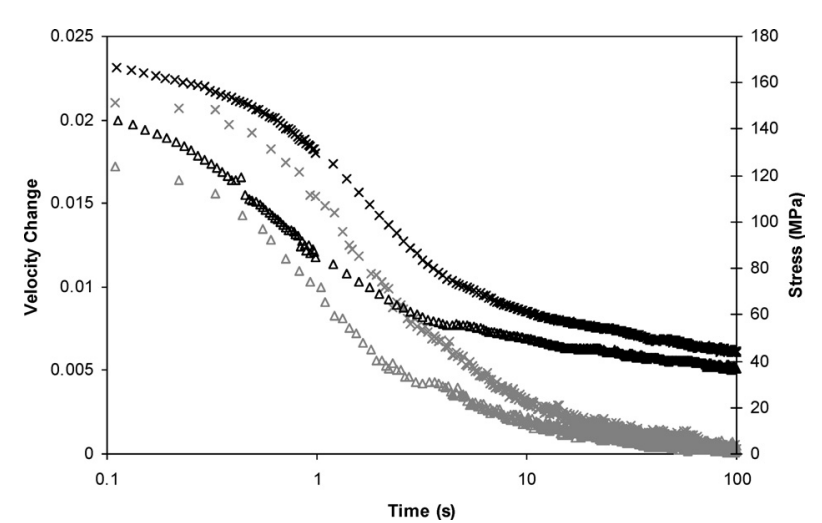

Fig. 7. Effect of temperature on ultrasonic velocity (in grey) and stress relaxation (in black). Crosses indicate $T=850^{\circ} \mathrm{C}$, whilst triangles indicate $T=900^{\circ} \mathrm{C}$. Strain $=0.5$, strain rate $=0.5 \mathrm{~s}^{-1}$.

high frequencies the experimental scatter is increased. The data collected at the frequency of $8 \mathrm{MHz}$ offers the best compromise between the above effects.

The attenuation curves shown in Fig. 6 can be split into three stages. Firstly, the attenuation appears to be approximately constant during the early stages of annealing. Secondly, when recrystallization starts there is a stronger decrease in attenuation. The rate of change in attenuation is smallest for the lower applied strain, again in agreement with the velocity data. In the third stage, approximately constant attenuation levels are obtained, although fluctuations are observed, particularly for the lowest strain, where the attenuation appears to increase slightly.

\subsection{Ultrasonics and Stress Relaxation: Effect of Tem- perature and Strain Rate}

The effects of temperature on ultrasonic velocity and stress relaxation is shown in Fig. 7. As can be seen, the stress relaxation rate and the rate of velocity change (defined by the time for $50 \%$ reduction) are higher in both cases for a temperature of $900^{\circ} \mathrm{C}$.

Figure 8 shows the effect of temperature on the ultrasonic attenuation. As can be seen, higher values of attenuation are observed for the temperature of $900^{\circ} \mathrm{C}$. In addition at this temperature, the rate of change in attenuation is highest, in agreement with the velocity and stress measurements.

Figure 9 shows that the rate of change in velocity is very similar for both strain rates. Likewise, the same effect is observed for the stress relaxation kinetics. For the attenuation results in Fig. 10 the values are higher for the lower strain rate of $0.1 \mathrm{~s}^{-1}$. In addition, the rate of change in attenuation is similar for both strain rates, again in agreement with the velocity and stress results.

\section{Discussion}

\subsection{Causes of Velocity Change and Attenuation}

Before embarking on a more quantitative analysis, we will first link observed changes in measured ultrasonic properties to known changes in the microstructure and dislocation density during each of the three phases: recovery, recrystallization and grain growth.

During recovery (the first stage) the texture and grain

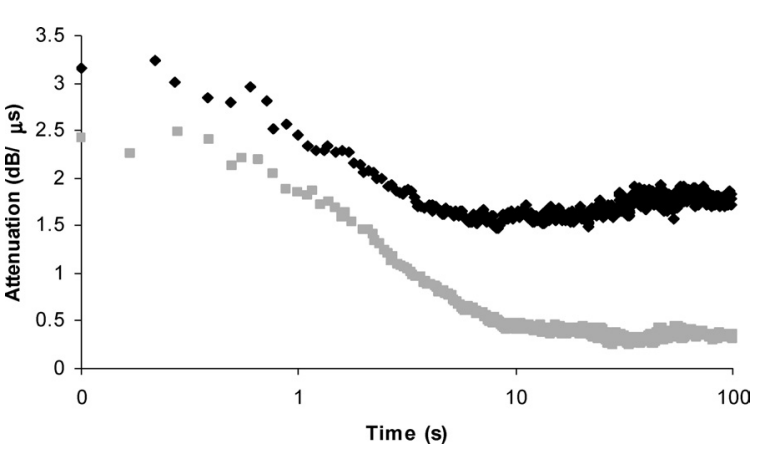

Fig. 8. Effect of temperature on ultrasonic attenuation at $8 \mathrm{MHz}$. Grey symbols indicate $T=850^{\circ} \mathrm{C}$, whilst black symbols indicate $T=900^{\circ} \mathrm{C}$. Strain $=0.5$, strain rate $=0.5 \mathrm{~s}^{-1}$.

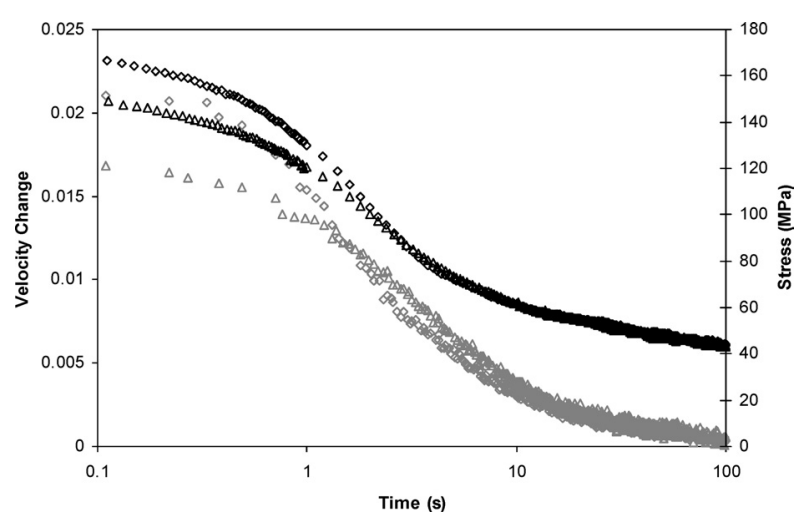

Fig. 9. Effect of strain rate on ultrasonic velocity (in grey) and stress relaxation (in black). Triangles indicate strain rate $=0.1 \mathrm{~s}^{-1}$, whilst diamonds indicate strain rate $=$ $0.5 \mathrm{~s}^{-1}$. Temperature $=850^{\circ} \mathrm{C}$, strain $=0.5$.

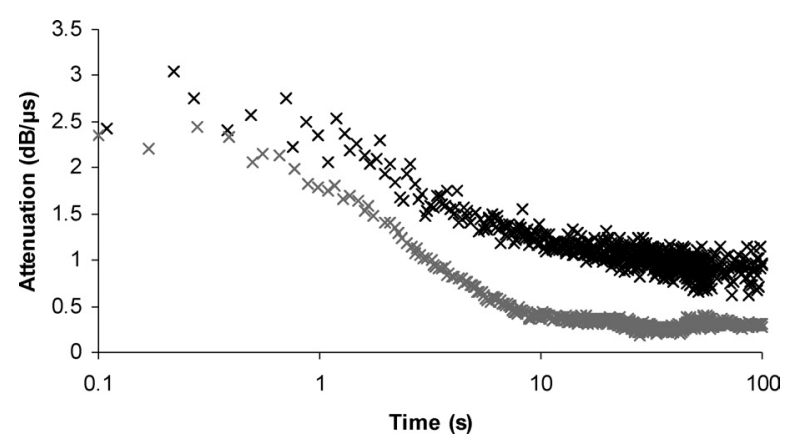

Fig. 10. Effect of strain rate on ultrasonic attenuation at $8 \mathrm{MHz}$. Black crosses indicate strain rate $=0.1 \mathrm{~s}^{-1}$, whilst grey crosses indicate strain rate $=0.5 \mathrm{~s}^{-1}$. Temperature $=$ $850^{\circ} \mathrm{C}$, strain $=0.5$.

size remain constant. Thus, from Eqs. (11) and (12) the observed slight decreases in velocity change and attenuation should be due to a small change in dislocation damping only. Assuming Eqs. (9) and (10) apply, this suggests that both $\rho L^{2}$ and $\rho L^{4}$ have decreased slightly. The dislocations that cause damping of the ultrasonic waves must be free to vibrate in response to the ultrasonic stress. ${ }^{18)}$ For the deformation conditions considered here most of the dislocations in austenite are expected to be immobile, i.e. arranged in cell walls or sub-grain boundaries and thus not able to vibrate. Therefore, the small change in damping is most likely due to a small number of mobile dislocations present in cell or sub-grain interiors.

During the second stage, where recrystallization is domi- 
nant, there is a rapid decrease in the total dislocation density (mobile and immobile). In addition both the texture and average grain size change.

During recrystallization, a decrease in the mobile dislocation density could influence the ultrasonic velocity change and attenuation through a change in dislocation damping. To estimate the magnitude of this effect the ultrasonic velocity change and attenuation can be calculated by applying Eqs. (9) and (10). For the velocity change, applying Eq. (9) with $\left.C \approx 0.5 G b^{2},{ }^{16}\right)$ and using estimated values for the mobile dislocation density $\left(10^{12} \mathrm{~m}^{-2}\right.$, as compared to a typical total dislocation density for the experiments of $\left.10^{14} \mathrm{~m}^{-2}\right)$ and for $L\left(10^{-7} \mathrm{~m}\right)$, gives an estimated initial velocity change of 0.0008 . Comparison of this value with the initial values in the experimental results, e.g. around 0.015 in Fig. 7, suggests that the dislocation damping effect accounts for only a small part of the observed velocity change during recrystallization, i.e. about $5 \%$. An estimate of the attenuation requires knowledge of the damping constant $B$ in austenite, but unfortunately no values for $B$ are available in the literature. Thus it cannot be said whether or not dislocation damping has a significant effect on the observed attenuation reduction during recrystallization.

During austenite recrystallization the overall texture is expected to weaken. ${ }^{23)}$ Thus, it can be derived from Eq. (1) that the velocity should change. However, it cannot unambiguously be said whether a weakening of texture will increase or decrease the ultrasonic velocity, since the parameter $W_{400}$ in Eqs. (1) and (2) can be either positive or negative.

During recrystallization the change in grain size is expected to influence the ultrasonic velocity and attenuation through the Eqs. (3) and (4). The exact effect of grain size is difficult to assess, since during recrystallization large deformed grains coexist with small recrystallizing grains, and their volume fractions change with time. Furthermore, Eqs. (3) and (4) have been derived for spherical grains. However, an indication of the maximum effect can still be made by calculations with Eqs. (3) and (4) for estimated initial average and final average grain sizes during recrystallization. Alternatively, an indication of the maximum effect can also be obtained from suitable experimental data in the literature. For the ultrasonic velocity, Palanichamy and co-work$\mathrm{ers}^{12)}$ have measured the ultrasonic velocity as a function of the grain size in austenitic stainless steel. The velocity was found to decrease with increasing grain size in an approximately linear fashion. Assuming that this behaviour also applies for austenite in this investigation, this allows the maximum effect of changing grain size to be estimated. For an applied strain of 0.5 , an estimated initial grain size of about $80 \mu \mathrm{m}$ and a recrystallized grain size of about $20 \mu \mathrm{m}$ (see Table 4), leads to an estimate for the maximum velocity change during recrystallization of around 0.0035 . Comparison with Fig. 7 indicates that this value is about $20 \%$ of the velocity change observed in this study. For the attenuation, Eq. (4) can be used to calculate the effect of grain size. Using a scattering factor of $1.3 \times 10^{-9} \mathrm{~s}^{3} \mathrm{~m}^{-3}$ at $850^{\circ} \mathrm{C}$ (see Sec. 5.3), with $f=8 \mathrm{MHz}$ and the same grain sizes as for the velocity calculation, gives an estimated maximum attenuation of $2.7 \mathrm{~dB} / \mu \mathrm{s}$ and a minimum of $0.04 \mathrm{~dB} / \mu \mathrm{s}$. As can be seen from e.g. Fig. 8, the experimental values fall
Table 3. Recrystallization start and finish times.

\begin{tabular}{|c|c|c|c|}
\hline Parameter & Strain & Rex start time (s) & Rex finish time (s) \\
\hline Stress & \multirow{3}{*}{0.2} & 2 & 40 \\
\hline Ultrasonic velocity change & & 5 & 35 \\
\hline Ultrasonic attenuation & & 3 & 18 \\
\hline Stress & \multirow[t]{3}{*}{0.35} & 1 & 22 \\
\hline Ultrasonic velocity change & & 1 & 18 \\
\hline Ultrasonic attenuation & & 2 & 33 \\
\hline Stress & \multirow[t]{3}{*}{0.5} & 0.4 & 10 \\
\hline Ultrasonic velocity change & & 0.5 & 11 \\
\hline Ultrasonic attenuation & & 0.8 & 8 \\
\hline
\end{tabular}

Table 4. Recrystallized grain sizes predicted from ultrasonics compared with values determined by optical microscopy.

\begin{tabular}{|c|c|c|}
\hline Strain & $\begin{array}{c}\text { Recrystallized grain } \\
\text { size from ultrasonics } \\
(\boldsymbol{\mu \mathbf { m } )}\end{array}$ & $\begin{array}{c}\text { Recrystallized grain size } \\
\text { from optical microscopy } \\
(\boldsymbol{\mu \mathbf { m } )}\end{array}$ \\
\hline 0.20 & 58 & 40 \\
\hline 0.35 & 28 & 25 \\
\hline 0.50 & 32 & 21 \\
\hline
\end{tabular}

between these two limits.

Thus, to conclude the second stage, the observed velocity change is expected to be controlled mostly by the texture change, since the other effects appear to account for a maximum of $25 \%$ of the total change. This conclusion is in agreement with work carried out by Kruger and coworkers $^{7)}$ on recrystallization of cold rolled IF steel, where it was found that the ultrasonic velocity remained approximately constant during recrystallization and only significantly increased when the texture was observed to change. The reduction in attenuation could be explained by a reduction in average grain size. Unfortunately the effect of dislocation damping could not be estimated.

In the third stage, where recrystallization is expected to be complete, growth of recrystallized grains should dictate the velocity change and attenuation. For the attenuation this is due to grain scattering. For the velocity change, in addition to being controlled by grain scattering, further changes in the overall texture during grain growth can also be a factor. However, since the velocity change and attenuation are approximately constant in this stage, this would indicate that the recrystallized grains do not grow significantly in size. This is in agreement with the experimental results on the grain size shown in Table 2 .

From the stress relaxation, ultrasonic velocity and attenuation results the start and finish times for recrystallization can be estimated by evaluation of the first and second slope changes. Results are shown in Table 3 for varying applied strain. As can be seen for the recrystallization start times, there is good agreement between times determined from stress, velocity and attenuation values. For the recrystallization finish times there is again good agreement between the stress and velocity values. For the attenuation results the agreement is less satisfactory.

\subsection{Comparison of Stress with Ultrasonic Velocity}

In order to compare stress relaxation and ultrasonic velocity results directly and to detect differences in sensitivity to one of the occurring microstructural changes, the fractional change in stress $F_{\sigma}$ and velocity $F_{\mathrm{V}}$ were calculated from: 


$$
\begin{gathered}
F_{\sigma}=\frac{\sigma_{t}-\sigma_{\mathrm{i}}}{\sigma_{\mathrm{f}}-\sigma_{\mathrm{i}}}, \\
F_{\mathrm{V}}=\frac{v_{t}-v_{\mathrm{i}}}{v_{\mathrm{f}}-v_{\mathrm{i}}},
\end{gathered}
$$

where $\sigma_{t}$ and $v_{t}$ are the values of stress and velocity at time $t, \sigma_{\mathrm{i}}$ and $v_{\mathrm{i}}$ are the initial values when $t=0.1 \mathrm{~s}$ and $\sigma_{\mathrm{f}}$ and $v_{\mathrm{f}}$ are the values after $100 \mathrm{~s}$ annealing. This choice of $v_{\mathrm{f}}$ and $\sigma_{\mathrm{f}}$ is expected to be similar in magnitude to $v_{\mathrm{f}}$ and $\sigma_{\mathrm{f}}$ obtained if the samples were annealed for much longer times, since in all experiments before or around $100 \mathrm{~s} v_{\mathrm{f}}$ and $\sigma_{\mathrm{f}}$ appear to approach their minimum values.

The fractional changes are plotted in Figs. 11-13 for strain, temperature and strain rate dependence experiments.

As can be seen, for varying temperature and strain rate there is little difference between the two measures of softening. However, when the strain is varied (Fig. 11), certain differences between the stress and ultrasonic values are observed for strains of 0.2 and 0.35 , with the magnitude of the difference being similar. At the highest strain there is no difference between the two measures of softening. As stated earlier, any differences observed should reflect the different sensitivities of the two techniques to recovery and recrystal-
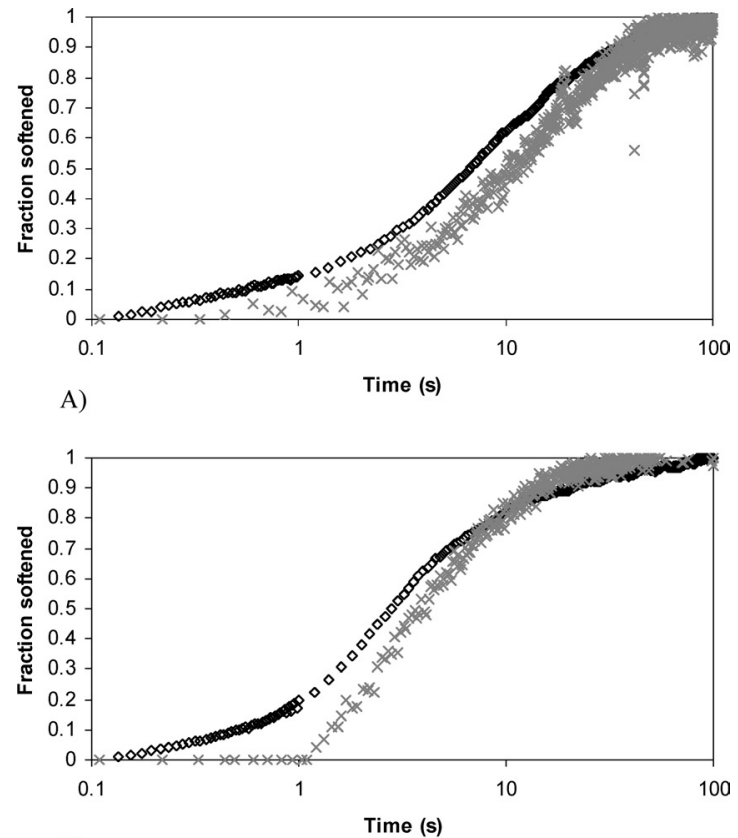

B)

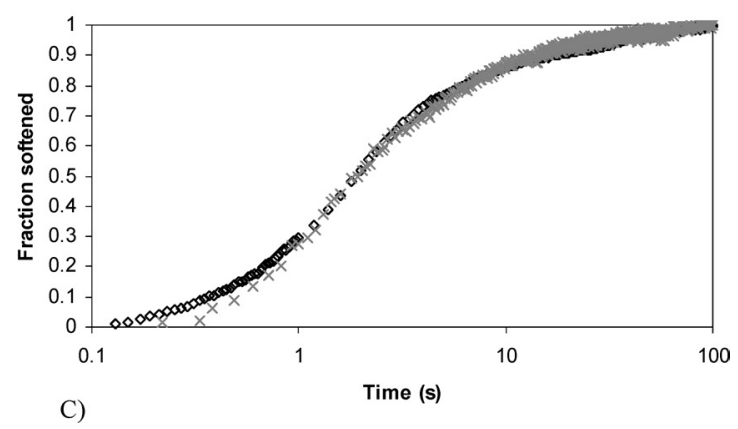

Fig. 11. Fractional change in stress (black diamonds) and velocity (grey crosses) for different applied strains. $T=850^{\circ} \mathrm{C}$, strain rate $=0.5 \mathrm{~s}^{-1}$. (A) Strain $=0.2$, (B) strain $=0.35$, (C) strain $=0.5$. lization. However, since recovery and recrystallization occurred in all experiments, the differences observed in Figs. 11(A) and 11(B) should also be apparent in Figs. 11(C), 12 and 13. Such differences are not observed, and therefore it is not possible to draw any conclusions regarding the different sensitivities of stress relaxation and ultrasonic measurements to recovery and recrystallization.

\subsection{Calculation of Recrystallized Grain Size}

Combining the attenuation data in Fig. 6 with data collected for other ultrasonic frequencies allows the attenua-
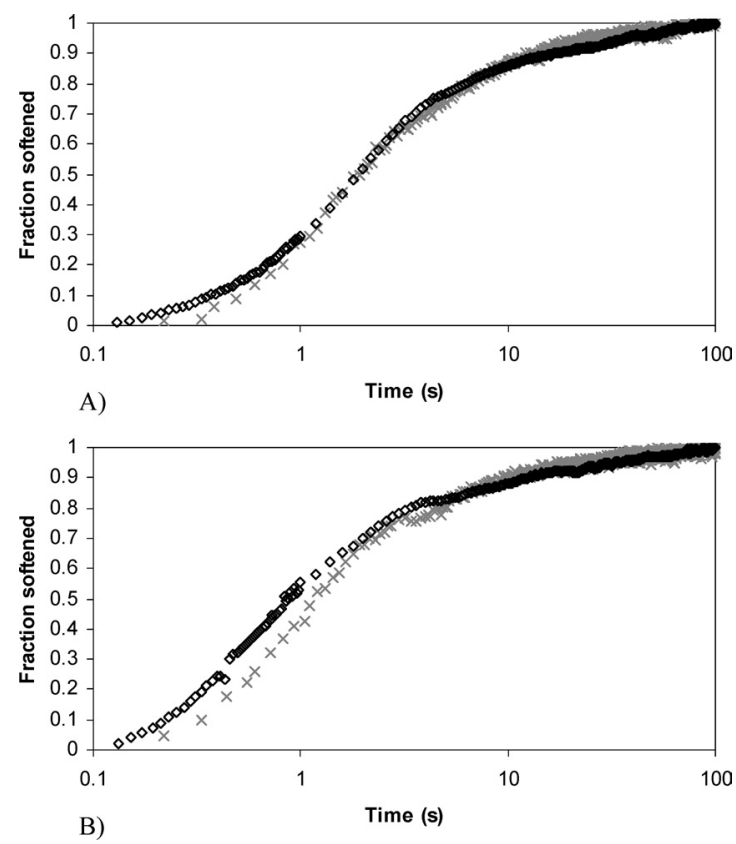

Fig. 12. Fractional change in stress (black diamonds) and velocity (grey crosses) for different temperatures. Strain $=0.5$, strain rate $=0.5 \mathrm{~s}^{-1}$. (A) $T=850^{\circ} \mathrm{C}$, (B) $T=900^{\circ} \mathrm{C}$.

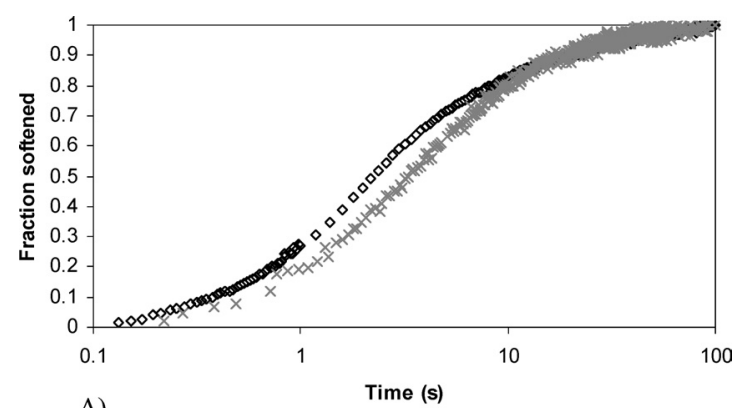

A)

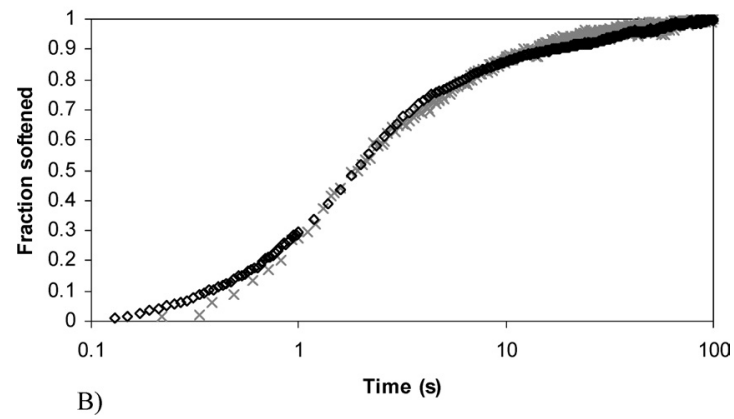

Fig. 13. Fractional change in stress (black diamonds) and velocity (grey crosses) for different strain rates. Strain $=0.5$, $T=850^{\circ} \mathrm{C}$. (A) Strain rate $=0.1 \mathrm{~s}^{-1}$, (B) strain rate $=$ $0.5 \mathrm{~s}^{-1}$ 

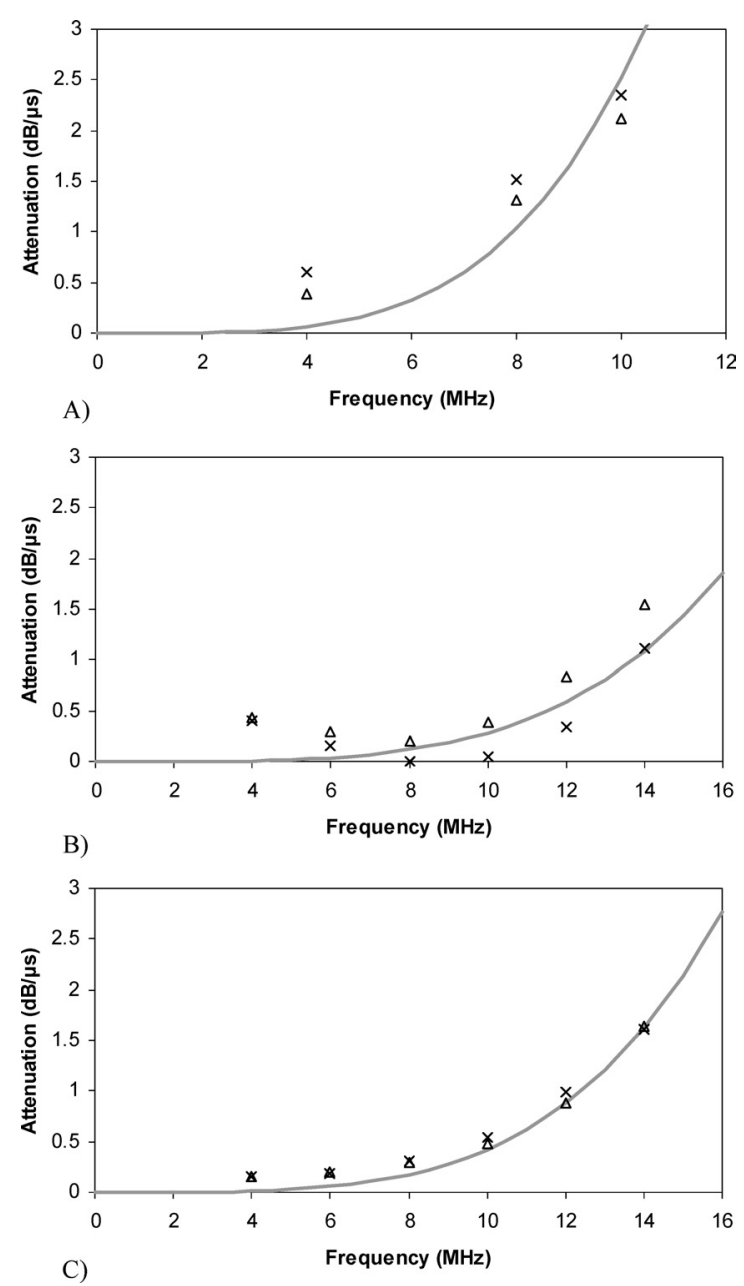

Fig. 14. Analysis of frequency effect on attenuation. $T=850^{\circ} \mathrm{C}$, strain rate $=0.5 \mathrm{~s}^{-1}$. (A) Strain $=0.2$, (B) $\mathrm{strain}=0.35$, (C) strain $=0.5$. Triangles indicate data for $t=50 \mathrm{~s}$ and crosses for $t=100 \mathrm{~s}$. Solid lines show best fit of Eq. (4) with experimental data.

tion at selected annealing times to be plotted as a function of frequency. This is shown for each of the three strains in Fig. 14. The two annealing times were chosen such that recrystallization was complete for all strains. Thus, the attenuation data should represent grain scattering by fully recrystallized grains only.

As can be seen from Fig. 14, the attenuation values are close together for the two annealing times. Since the attenuation depends on the third power of the grain size, the data suggests that there is no significant grain growth between 50 and $100 \mathrm{~s}$ annealing for each strain. This is in agreement with the grain size data obtained by optical microscopy (Table 2).

Using Eqs. (4), (7) and (8) the recrystallised grain size can be estimated, provided that the single crystal elastic constants, longitudinal and shear velocities are known. As these values are temperature dependent, values corresponding to the temperature of interest, i.e. $850^{\circ} \mathrm{C}$, must be chosen. Regretfully, accurate data on the elastic constants for $\mathrm{C}-\mathrm{Mn}$ steels in the austenitic state do not exist. Instead literature data for the temperature dependence of the elastic constants in $\mathrm{Fe}-30 \mathrm{wt} \% \mathrm{Ni}$ single crystal have been used. ${ }^{24)}$ This alloy has been shown to be a good model alloy in representing the mechanical properties of austenite in carbon steels. $^{25,26)}$

To calculate the scattering factor, $v_{\mathrm{L}}$ was taken from the experimental data at $850^{\circ} \mathrm{C}$ (after recrystallization) as $4970 \mathrm{~m} / \mathrm{s}$, whilst $v_{\mathrm{s}}$ was assumed to be $0.5 v_{\mathrm{L}}$. The elastic constants for the $\mathrm{Fe}-30 \mathrm{wt} \% \mathrm{Ni}$ alloy at $850^{\circ} \mathrm{C}$ were obtained by extrapolation of the original data set in Ref. 24). This gave $c_{11}=177 \mathrm{GPa}, c_{12}=130 \mathrm{GPa}$ and $c_{44}=95 \mathrm{GPa}$. With these parameter values $S_{\mathrm{L}}$ was calculated to be $1.3 \times 10^{-9} \mathrm{~s}^{3} / \mathrm{m}^{3}$. Using the grain size as a fitting parameter, the attenuation can be calculated as a function of frequency. The resulting dependence is indicated in Fig. 14 by solid lines. As can be seen there is reasonable agreement with experimental data for the strains of 0.35 and 0.5 . For the strain of 0.2 the agreement is less satisfactory, although there are very few data points.

The calculated recrystallised grain sizes are shown in Table 4, together with the values determined by optical microscopy. As can be seen, the grain size is largest for the lowest strain as expected. However, for the strain of 0.35 the grain size is slightly smaller than that at a strain of 0.5 . This is attributed to experimental error in the attenuation measurements.

Comparison of the calculated grain sizes for each strain with the corresponding values determined optically, shows generally reasonable quantitative agreement considering the assumptions made and the accuracy of the attenuation measurements. The largest difference in grain size was obtained for the lowest applied strain $(18 \mu \mathrm{m})$.

An alternative to obtaining grain sizes by confronting experimental attenuation with modelled attenuation, could be to use an experimentally obtained relationship between attenuation and austenite grain size in the literature. ${ }^{10)}$ However, this relationship was determined for a single frequency $(15 \mathrm{MHz})$ and for higher temperatures $\left(1100^{\circ} \mathrm{C}\right)$ than used here. Since the calibration constant is temperature dependent, attempting to extrapolate the relation to lower temperatures would be highly dubious.

Finally, a factor to be considered is the strain distribution in the sample due to friction at the work-tool sample interfaces. The grain size detected by ultrasonic waves represents an average across the sample diameter at the centre of each specimen. The grains assessed by optical microscopy were those only in the centre of the specimens. However, FEM simulations for the current experimental conditions have shown that for the applied strain with the largest variation in strain distribution (0.5), the strain in the centre of the samples $(0.65)$ extends through $60 \%$ of the sample diameter at the centre. In addition, the remaining $40 \%$ of the diameter is at a strain of around 0.5 . The average strain of these two regions is 0.59 , which will control the grain size detected by ultrasonics. Thus, since the average strain of 0.59 is close to that of the centre $(0.65)$, the average grain size "experienced" by ultrasonic waves should be similar to the grain size seen in the centre of the specimens by optical microscopy.

Overall, despite the many challenges to ultrasonically obtain accurate quantitative grain sizes, a simple observation of the obtained attenuation shows unmistakably the qualitative picture of grain size, i.e. a significantly higher attenuation (larger grains) being observed for a strain of 0.2 compared to similar and smaller attenuations (smaller grains) 
being observed for the strains of 0.35 and 0.5 .

\section{Conclusions}

(1) For the first time, the laser-ultrasonics technique has been used to characterize the recovery and recrystallization processes of hot deformed austenite in-situ.

(2) During recovery preceding recrystallization the ultrasonic velocity change and attenuation decreased only slightly. This was explained in terms of the ultrasonic waves interacting with only a small number of mobile dislocations present in the deformed austenite.

(3) The observed decrease in velocity change during recrystallization was mostly due to a change in the overall texture. The decrease in attenuation during recrystallization could be explained by a decrease in grain size, whilst the effect of dislocation damping could not be estimated.

(4) When recrystallization was complete the ultrasonic measurements indicated little or no grain growth, which agreed with observations by optical microscopy.

(5) The recrystallization kinetics as revealed by stress relaxation, ultrasonic velocity and attenuation for a variety of deformation and temperature conditions, were generally in good agreement.

(6) Qualitatively the attenuation indicated clear differences in grain size for the tested samples. Calculated recrystallized grain sizes from the ultrasonic attenuation showed generally reasonable agreement with values obtained by optical microscopy.

\section{Acknowledgements}

Thanks to the IMI-National Research Council of Canada, for allowing us to perform these experiments and to $\mathrm{M}$. Lord, in particular for the experimental assistance. Thanks also to T. Zuidwijk for technical support concerning the Gleeble $^{\circledR} 3500$. Finally thanks to Professor M. Militzer for useful discussions during his sabbatical stay in Delft.

\section{REFERENCES}

1) A. Smith, H. Luo, D. N. Hanlon, J. Sietsma and S. van der Zwaag: ISIJ Int., 44 (2004), 1188.

2) L. P. Karjalainen: Mater. Sci. Technol., 11 (1995), 557.

3) L. P. Karjalainen and J. Perttula: ISIJ Int., 36 (1996), 736.

4) H. Luo, J. Sietsma and S. van der Zwaag: Metall. Mater. Trans. A, 35A (2004), 1889.
5) S. Kruger, A. Moreau, M. Militzer and T. Biggs: THERMEC'2003, Proc. Int. Conf. on Processing and Manufacture of Advanced Materials, ed. by T. Chandra, J. Torralba and T. Sakai, (Materials Science Forum 426-432), Trans Tech Publications, Zürich, Switzerland, (2003), 483.

6) C. Scruby, C. Bull, R. Young and F. Humphreys: Mater. Sci. Technol., 19 (2003), 163.

7) S. Kruger, G. Lamouche, A. Moreau and M. Militzer: Materials Science and Technol 2004 Conf. Proc. (CD only), AIP, New York, (2003), 809

8) E. Ulmgren, M. Ericsson, D. Artymowicz and B. Hutchinson: Proc. Int. Conf. on Recrystallization and Grain Growth, ed. by B. Bacroix, J. Driver, R. Le Gall, C. Maurice, R. Penelle, H. Regle and L. Tabourot, (Materials Science Forum 467-470), Trans Tech Publications, Zürich, Switzerland, (2004), 1353.

9) S. Sundin and D. Artymowicz: Metall. Mater. Trans. A, 33A (2002), 687.

10) M. Dubois, M. Militzer, A. Moreau and J. Bussiere: Scr. Mater, 42 (2000), 867.

11) M. Dubois, A. Moreau, M. Militzer and J. Bussiere: Scr. Mater, 39 (1998), 735.

12) P. Palanichamy, A. Joseph, T. Jayakumar and B. Raj: NDT \& E Int., 28, (1995), 179.

13) B. Hutchinson, B. Moss, A. Smith, A. Astill, C. Scruby, G. Engberg and J. Bjorklund: Ironmaking Steelmaking, 29 (2002), 80.

14) A. Moreau, D. Levesque, M. Lord, M. Dubois, J. Monchalin, C. Padioleau and J. Bussiere: Ultrasonics, 40 (2002), 1047.

15) G. Jeskey, R. Kolarik, II, E. Damm, J. Monchalin, G. Lamouche, S. Kruger and M. Choquet: Materials Science and Technology Conf. Proc., 1, AIST (Association for Iron \& Steel Technology) and TMS (The Minerals, Metals \& Materials Society), (2004), 823.

16) M. Hirao, H. Ogi, N. Suzuki and T. Ohtani: Acta, Mater, 48 (2000), 517.

17) S. Kenderian, T. Berndt, R. Green, Jr. and B. Djordjevic: Mater. Sci. Eng. A, A348 (2003), 90 .

18) T. Ohtani, H. Ogi and M. Hirao: Metall. Mater. Trans. A, 36A (2005), 411 .

19) H. Ogi, H. Ledbetter and S. Kim: Metall. Mater. Trans. A, 32A (2001), 1671.

20) S. Hirsekorn: J. Acoust. Soc. Am., 72 (1982), 1021.

21) E. Papadakis and E. Reed: J. Appl. Phys., 32, (1961), 682.

22) A. Granato and K. Lucke: J. Appl. Phys., 27, (1956), 583.

$23)$ B. Verlinden, Ph. Bocher, E. Girault and E. Aernoudt: Scr. Mater, 45 (2001), 909

24) G. Alers, J. Neighbours and H. Sato: J. Phys. Chem. Solids, 13 (1960), 40.

25) F. Bai, P. Cizek, E. Palmiere and W. Rainforth: Proc. Int. Conf. on Recrystallization and Grain Growth, ed. by B. Bacroix, J. Driver, R. Le Gall, C. Maurice, R. Penelle, H. Regle and L. Tabourot, (Materials Science Forum 467-470), Trans Tech Publications, Zürich, Switzerland, (2004), 21.

26) P. Cizek, F. Bai, W. Rainforth and J. Beynon: Mater. Trans., 45 (2004), 2157. 